\title{
Complex and dynamic population structures: synthesis, open questions, and future directions
}

\author{
Joshua L. Payne • Mario Giacobini • \\ Jason H. Moore
}

Published online: 6 February 2013

(c) Springer-Verlag Berlin Heidelberg 2013

\begin{abstract}
The population structure of an evolutionary algorithm influences the dissemination and mixing of advantageous alleles, and therefore affects search performance. Much recent attention has focused on the analysis of complex population structures, characterized by heterogeneous connectivity distributions, non-trivial clustering properties, and degree-degree correlations. Here, we synthesize the results of these recent studies, discuss their limitations, and highlight several open questions regarding (1) unsolved theoretical issues and (2) the practical utility of complex population structures for evolutionary search. In addition, we will discuss an alternative complex population structure that is known to significantly influence dynamical processes, but has yet to be explored for evolutionary optimization. We then shift our attention toward dynamic population structures, which have received markedly less attention than their static counterparts. We will discuss the strengths and limitations of extant techniques and present open theoretical and experimental
\end{abstract}

Communicated by E. Alba.

\section{J. L. Payne $(\bowtie)$}

Institute of Evolutionary Biology and Environmental Studies, University of Zurich, Building Y27-J-48, Winterhurerstrasse 190, Zurich, CH 8057, USA

e-mail: joshua.payne@ieu.uzh.ch

\section{Giacobini}

Department of Veterinary Sciences and Molecular

Biotechnology Center, University of Torino, Torino, Italy

e-mail: mario.giacobini@unito.it

\section{J. H. Moore}

Computational Genetics Laboratory, Dartmouth Medical School,

1 Medical Center Drive, Lebanon, NH, USA

e-mail: Jason.H.Moore@Dartmouth.edu questions and directions for future research. In particular, we will focus on the prospects of "active linking," wherein edges are dynamically rewired according to the genotypic or phenotypic properties of individuals, or according to the success of prior inter-individual interactions.

Keywords Assortativity - Evolutionary algorithms . Interaction topologies - Networks · Scale-free ·

Small-world

\section{Introduction}

There are several design choices that must be made when applying an evolutionary algorithm to an optimization task. The genotypic and phenotypic representations, variation operators and probabilities, and selection scheme must all be chosen carefully to ensure the algorithm is well-suited for the application. One design choice that is often overlooked is the population structure, which specifies the network of potential interactions (e.g., mating, competition) between individuals in the population. The vast majority of research on evolutionary algorithms has focused on the well-mixed population, also referred to as panmixia, in which every individual has the potential to interact with every other individual. Some of the most fundamental results in the field, including the schema theorem (Holland 1975), are built atop the assumption of a panmictic population.

The study of non-panmictic populations was born in the context of parallelization, with the goal of improving algorithm efficiency (Cohoon et al. 1987; Grefenstette 1981; Tanese 1987). Populations were distributed across several computing cores and a communication network was established that specified how individuals could be exchanged between cores. These studies quickly led to the 
insight that population structure could not only improve algorithm efficiency, but also search efficacy (GorgesSchleuter 1989; Manderick and Spiessens 1989). By limiting the scale of interaction events, population structure implicitly reduced selective pressure and enhanced the explorative properties of evolutionary search (Giacobini et al. 2005b). Population structure then became its own object of study, independent of parallelization, and the subfield of cellular evolutionary algorithms (CEAs) was established (for a comprehensive history of CEAs, please see Alba and Tomassini (2002)).

CEAs are now a mature subfield of evolutionary computation, with two well-known books on the topic (Alba and Dorronsoro 2008; Tomassini 2005) and dozens of research articles. The primary focus of the field has been on static regular lattices, in which every individual has a fixed number of potential interaction partners and this number remains constant throughout the evolution of the population. Two fundamental observations that can be drawn from this line of research are that selective pressure (1) is a function of readily computable topological properties of the population structure (Sarma and De Jong 1996; Rudolph 2000) and (2) is generally reduced in lattice-based population structures, relative to panmixia (Giacobini et al. 2005b).

Over the past decade, there has been an intense research effort to characterize the interaction patterns of real-world populations (e.g., Barabàsi and Albert (1999); Liljeros et al. (2001); Onnela et al. (2007); Watts and Strogatz (1998)). These studies have uncovered several structural properties that deviate substantially from those observed in both latticebased population structures and panmictic populations. For example, heterogeneous degree distributions (Barabàsi and Albert 1999), dynamic edge weights (Goldbeck et al. 2003), non-trivial clustering properties (Watts and Strogatz 1998), and degree-degree correlations (Newman 2002) are commonly observed. The implications of these structural properties for dynamical processes, such as the spread of biological (Boguñá et al. 2003; Pastor-Satorras and Vespignani 2001) and social contagion (Payne et al. 2009; Watts 2002) and the evolution of cooperation (Rong et al. 2007; Santos and Pacheco 2005), were quickly realized thereafter. However, the implications of using such complex networks as population structures in CEAs are only beginning to be appreciated. The goal of this article is to synthesize the existing theoretical and experimental results on complex and dynamic population structures, to highlight open questions, and to pose potential directions for future research in the area.

\subsection{Network terminology}

We begin with a brief presentation of the terminology used in this review. For a comprehensive introduction to networks and their nomenclature, the reader is referred to Newman (2010).

Networks are made up of vertices and edges. In CEAs, they are used to define the structure of interactions between individuals in the population. Each individual occupies a single vertex and the terms individual and vertex can therefore be used interchangeably. Vertices that share an edge are referred to as neighbors. The neighborhood of a vertex consists of all vertices with which that vertex shares an edge. All inter-individual interactions occur at the level of the neighborhood. These interactions are symmetric, so the edges are undirected. The number of vertices in a neighborhood is the vertex degree. If all vertices have the same degree, then the network is regular, otherwise it is irregular. All complex networks are irregular. One fundamental property of irregular networks is the degree distribution, which describes the probability $p_{k}$ that a vertex is of degree $k$. The first moment of this distribution provides the average degree $z$; the first inverse moment provides a weighted average of the inverse degrees. Other important properties are (1) clustering, a metric that captures the frequency of closed triangular motifs in a network (Newman 2003); (2) characteristic path length, a measure of the average all-pairs shortest distance between vertices (Watts and Strogatz 1998); and (3) degree-degree assortativity, which captures the propensity with which vertices of similar degree attach to one another (Newman 2002).

Three classes of complex population structures have been explored for use in CEAs, which vary in their topological properties and methods of construction. Random networks are often generated by placing a fixed number of edges between vertices at random with uniform probability (Erdős and Rényi 1959). They possess a Poisson degree distribution $p_{k}=z^{k} \mathrm{e}^{-z} / k$ !, a short characteristic path length, and a vanishingly small clustering coefficient. Small-world networks are often generated by probablistically rewiring the edges of a regular network (Watts and Strogatz 1998). For small edge-rewiring probabilities, they possess a degree distribution that deviates slightly from a Dirac delta function, a short characteristic path length, and a high clustering coefficient. Scale-free networks are often generated with the preferential attachment method, in which the network is built incrementally with new vertices attaching to existing vertices in proportion to their degree (Barabàsi and Albert 1999). They possess a degree distribution that follows a power-law $p_{k} \propto k^{-\gamma}$, where $\gamma$ is referred to as the scaling parameter. Their other topological properties, such as characteristic path length, clustering coefficient, and assortativity depend on the algorithm used to construct the network. 


\subsection{Panmictic populations}

The traditional panmictic population structure can also be formalized as a network, wherein every individual is connected to every other individual. Panmictic population structures are therefore complete graphs. As such, the degree distribution follows a Dirac delta function and both the assortativity and clustering coefficient take on their maximum values of one.

\section{Complex population structures}

\subsection{Synthesis of theoretical results}

Complex population structures are those that exhibit nontrivial topological properties, which deviate from those of regular and random population structures. Most theoretical analyses of complex population structures have focused on the relationship between the topological properties of interaction networks and selective pressure. One useful method for quantifying the influence of population structure on selective pressure is takeover time analysis (Goldberg and Deb 1991). Takeover time is defined as the expected number of generations required for a single advantageous allele to fully saturate a population, in the absence of variation operators such as recombination and mutation. Takeover time and selective pressure are inversely related, such that higher takeover times suggest lower selective pressure and vice versa.

Giacobini et al. (2005a) provided the seminal analysis of takeover time in complex population structures, developing mathematical models to describe the selective pressure induced by random and small-world population structures, and conducting simulations of takeover dynamics in scale-free population structures. The main conclusions of their study can be summarized as follows. Random population structures induced a selective pressure commensurate with panmictic populations, which increased with increasing average degree. This led to the practical conclusion that it may not be necessary to consider the entire population for interaction events, as done in panmictic populations, because the random population structure exhibits similar behavior using only a fraction of the interactions.

Selective pressure in small-world population structures increased as the probability of edge rewiring increased. As this probability was tuned from zero toward one, selective pressure rapidly shifted from very low values, as induced by ring-based population structures, to very high values, as induced by random and panmictic population structures. This rapid transition was attributed to the correspondingly rapid decrease in the population structure's characteristic path length. Thus, the rewired edges provided shortcuts in the network of inter-individual interactions, allowing for the more rapid dissemination of advantageous alleles. Similar results are obtained when edges are randomly added, rather than rewired (Dorronsoro and Bouvry 2012).

The selective pressures induced by scale-free population structures were also considered by Giacobini et al. (2005a), using interaction networks generated with the well-known preferential attachment algorithm (Barabàsi and Albert 1999). Average takeover times were again quantitatively similar to those observed in random and panmictic population structures, indicating that these three classes of population structures induced comparable selective pressures. However, when the initial high-fitness allele was placed in a high-degree vertex, takeover time was dramatically decreased, leading the authors to conclude that "scale-free graphs are a topology in which propagation is at least as fast as for random graphs."

The generality of this statement was put to the test in a follow-up study by Payne and Eppstein (2007). By considering a variety of algorithms for generating scale-free population structures, it was shown that selective pressure could vary from high levels, comparable to those induced by random mixing, to low levels that were even weaker than those induced by a two-dimensional toroidal lattice with $3 \times 3$ square neighborhoods of interactions (i.e., Moore neighborhoods). Thus, knowing only that a population structure has a power-law degree distribution is not sufficient for quantifying the selective pressure it induces on a population. The exact form of the power-law, plus additional topological properties, play an important role.

To better characterize the influence of these topological properties on selective pressure, Payne and Eppstein (2008, 2009a) subsequently considered a broader class of scalefree population structures, in which two topological properties were systematically varied: (1) degree-degree assortativity $r$ and (2) the scaling exponent $\gamma$ of the degree distribution $p_{k} \propto k^{-\gamma}$, which captures in part the heterogeneity in the distribution of vertex connectivity. These properties were found to affect the rate of flow of advantageous alleles and therefore affect selective pressure. Takeover time exhibited a positive correlation with $r$, and a negative correlation with $\gamma$, with additional nonlinear interactions between these two quantities. This indicates that selective pressure is minimized when the scale-free population structure is both assortative (high $r$ ) and degreeheterogeneous (low $\gamma$ ). The variability in takeover times between individual placements of the initial advantageous allele were also influenced by these topological properties. Specifically, this variability increased with decreasing $\gamma$ and was highest for disassortative $(r<0)$ interaction networks (Payne and Eppstein 2009a). In assortative interaction networks $(r>0)$, this variability was relatively 
reduced, and takeover times were essentially independent of the degree of the vertex in which the initial advantageous allele was placed (Payne and Eppstein 2008), in contrast to the strong negative correlation observed in disassortative and uncorrelated (Giacobini et al. 2005a) scale-free population structures. Thus, the ability of highly connected vertices to act as "selection amplifiers" (Lieberman et al. 2001; Whigham and Dick 2008a) is dependent upon degree-degree correlations.

A central goal of theoretical work on complex population structures is to derive a general and broadly applicable functional relationship between the topological properties of a population structure and the selective pressure it induces on a population. Such a relationship has been obtained for one-dimensional (Rudolph 2001) and twodimensional (Sarma and De Jong 1996) regular population structures, the latter of which has found practical application in approximating takeover dynamics analytically (Payne and Eppstein 2009b) and dynamically restructuring lattice-based population structures to improve the search performance of genetic algorithms (Alba and Dorronsoro 2005). The analogous relationship for complex population structures has proven more elusive. Payne and Eppstein (2007, 2008, 2009a) have made significant progress in this direction by determining that selective pressure in scalefree population structures can be accurately approximated as a planar function of the average inverse degree and the population size. However, this functional relationship is far from all-encompassing. Even for scale-free population structures, it is of limited use because it does not take into account the effects of assortativity. Further, the average inverse degree cannot differentiate between a toroidal lattice and a regular random graph of the same average degree, and would therefore predict that these two population structures induce the same selective pressure when it is well known that they do not (Giacobini et al. 2005a). The search for a universal relationship between topology and selective pressure is therefore ongoing.

Another technique for analyzing the influence of population structure on selective pressure is to differentiate between the topology of potential interactions and the topology of actual interactions, either within a single generation (Payne and Eppstein 2006) or across generations (Whigham and Dick 2008b; Whitacre et al. 2009). If in either case the degree distribution is heavily skewed (e.g., following a power law), then a minority of individuals are taking part in the majority of interactions, and their genetic material can be expected to dominate the population. The main advantage of this form of analysis is that it is independent of the specifics of the evolutionary algorithm, which makes the technique broadly applicable. Further, the analysis includes the effects of recombination, a variation operator that is central to many evolutionary algorithms, but is not included in takeover time analysis.

Whitacre et al. (2009) developed a technique that encoded a subset of an individual's genealogical history as a directed network, tracing the flow of genetic information across generations. Topological analysis of these networks revealed that power-law degree distributions were a common outcome, indicating that a small number of individuals had a dominating effect on the evolutionary dynamics of a population. However, when the population was structured as a ring, Whitacre et al. (2009) noted a deviation from the power-law degree distribution in the genealogical networks, providing further evidence that such regular population structures reduce selective pressure.

Analysis of networks of recombination events within a generation has revealed similar results. Payne and Eppstein (2006) characterized the mating topologies of a standard genetic algorithm used to solve a benchmark optimization problem, where within each generation an edge was drawn between two individuals if they took part in a recombination event. This study considered a variety of population structures, including panmixia, toroidal lattices with various neighborhood sizes, small-world networks with various rewiring probabilities, and uncorrelated $(r \approx 0)$ scale-free networks with $\gamma \approx 3$. Across the vast majority of these population structures, the degree distributions of the mating topologies followed a power-law. This indicates that only a few individuals took part in the majority of recombination events, regardless of the underlying population structure. The only exception to this trend occured when using a lattice-based population structure with nearest-neighbor interactions. Thus, the analogous observation made by Whitacre et al. (2009) that genealogical networks are often power-law distributed may stem directly from the connectivity distributions of the mating topologies observed within each generation (Payne and Eppstein 2006). Unsurprisingly, a recent analysis of the combined topologies of mating interactions and genealogical history has also found power-law behavior (Jieyu et al. 2012).

An interesting distinction can be made between the power-law distributed mating topologies observed on scale-free and panmictic population structures (Payne and Eppstein 2006). In the former case, there is initially no correlation between an individual's fitness and its degree in the mating topology, whereas in the latter case there is a strong correlation. Taking this observation together with those made by Whitacre et al. (2009), the quality of the genetic material that dominates a population over evolutionary time is directly related to the topological properties of the underlying population structure. Thus, while scalefree population structures often lead to the rapid dissemination of alleles (Giacobini et al. 2005a; Payne and 
Eppstein 2007, 2008, 2009a), the content of this genetic information has the potential to lead the population astray.

\subsection{Open theoretical questions}

Despite the many theoretical advances outlined in the previous section, open questions and outstanding challenges abound:

1. How do complex population structures affect the fixation probabilities of advantageous alleles?

The majority of theoretical analyses of complex population structures have focused on the dissemination of genetic material. These analyses assume that advantageous alleles cannot be outcompeted and therefore implicitly include elitism. A complete theoretical understanding of complex population structures requires the relaxation of this assumption. Significant progress has been made in this direction by Whigham and Dick (2008a) for ring and star topologies, but the extension to complex population structures has yet to be carried out. An additional extension would be to relax the non-extinction assumption (Rudolph 2001), so that advantageous alleles can not only be outcompeted, but can also be lost via reverse mutation. This is analogous to a recovery event in an epidemiological model (Payne and Eppstein 2009b).

2. How do complex population structures affect the mixing of genetic material?

Takeover time analysis provides useful information regarding the influence of population structure on selective pressure. However, it ignores the effects of recombination, which is the primary variation operator in many evolutionary algorithms. It is therefore of crucial importance to understand how complex population structures affect the mixing of genetic material. Such an investigation has been performed for panmictic genetic algorithms (Thierens and Goldberg 1993), but its extension to complex population structures has yet to be undertaken.

3. Develop analytical models of takeover dynamics in scale-free population structures.

While analytical models of takeover dynamics exist for regular, random, and small-world population structures (Giacobini et al. 2005a, b; Rudolph 2000), there is no corresponding model for scale-free population structures. The development of such a model could further elucidate the relative influences of scaling $(\gamma)$ and assortativity $(r)$ on selective pressure and the relationship between vertex degree and expected saturation time. Analytical models of alternative spreading processes on scale-free networks may provide a useful frame of reference (e.g., Pastor-Satorras and Vespignani (2001); Sood et al. (2008)).
4. Derive a universal functional relationship between selective pressure and the topological properties of complex population structures.

As previously mentioned, direct functional relationships between selective pressure and the topological properties of regular population structures have been obtained (Sarma and De Jong 1996; Rudolph 2000). For example, selective pressure in an arbitrary two-dimensional, lattice-based population structure is a function of the ratio of the radius of the local neighborhood to the radius of the global lattice (Sarma and De Jong 1996). Since the radius metric is a function of the Euclidean distance between vertices, it cannot be applied to most complex population structures, as these are typically not embedded in Cartesian space. The derivation of an analogous metric for complex population structures would allow for the rapid assessment of selective pressure from readily computable topological properties, and preclude the need to run expensive computational simulations.

\subsection{Synthesis of experimental results}

Experimental studies that used complex population structures for evolutionary optimization began to appear immediately after the first theoretical analysis by Giacobini et al. (2005a). While the theory was continuing to develop (Payne and Eppstein 2006, 2007, 2008, 2009a; Whigham and Dick 2008a, b; Whitacre et al. 2009), several investigations put complex population structures directly into practice (DeFelice et al. 2011; Foo and Kirley 2008; Gasparri et al. 2007, 2009; Giacobini et al. 2006; Kirley and Stewart 2007a; Kirley and Stewart 2007b; Min et al. 2006). As such, many of the experimental observations were not couched in terms of theoretical results, because they were not yet known. In this section, we discuss these experimental studies and place them into the context of known theory, where possible.

Giacobini et al. (2006) provided the first experimental results for small-world population structures, applying a mutation-based genetic algorithm to a variety of benchmark optimization problems. These population structures were constructed in the same manner as in the earlier theoretical work of Giacobini et al. (2005a), allowing for the direct comparison between theory and experiment. Small-world population structures led to higher success rates on three out of the four optimization problems analyzed, but came at the expense of an increased number of required evaluations, relative to panmictic populations. However, the number of required function evaluations is often fewer than that required by grid-based CEAs, while still offering competitive solution quality (Dorronsoro and Bouvry 2012). These observations are in line with the 
theoretical results for selective pressure in small-world population structures, where low probabilities of edgerewiring led to high takeover times and thus low selective pressure (Giacobini et al. 2005a). Similar observations were made by Min et al. (2006) and later DeFelice et al. (2011), who noted a decrease in genetic diversity as the population structure was rewired from a regular lattice toward a random network. This effect can be attributed to the increase in selective pressure that accompanies an increase in the probability of edge-rewiring (Giacobini et al. 2005a). In contrast to Giacobini et al. (2006), a degradation in search performance was observed by DeFelice et al. ((2011) on a multimodal combinatorial benchmark, where the number of global optima maintained by the population dramatically decreased as the probability of edge rewiring increased. Similarly, Foo and Kirley (2008) found a degradation in search performance on epistatic optimization benchmarks as the probability of edge rewiring increased, an observation they attributed to a decrease in clustering. However, the random rewiring of edges not only affects clustering, but also characteristic path length, a topological property that is strongly correlated with selective pressure in both regular (Rudolph 2000; Payne and Eppstein 2007) and small-world (Giacobini et al. 2005a) population structures. As the two quantities are confounded, the independent effects of clustering on the search performance of structured genetic algorithms remains to be seen.

Giacobini et al. (2006) also provided the first experimental results for scale-free population structures and found that they were always outperformed by panmictic populations, both in terms of success rate and convergence speed. These results are also consistent with theory. As the networks were generated according to the preferential attachment method (Barabàsi and Albert 1999), they were uncorrelated $(r \approx 0)$ with a scaling exponent of $\gamma \approx 3$, indicating high selective pressure and the propensity for premature convergence (Giacobini et al. 2005a; Payne and Eppstein 2009a).

Gasparri et al. (2007, 2009) used a genetic algorithm with a scale-free population structure to solve the localization and kidnap problems in a mobile robotics application. The authors claimed improved solution quality on both tasks and attributed these improvements to the creation and maintenance of genotypic niches. However, for a population structure to promote niching it must induce a low selective pressure, and this only occurs for a specific subset of scale-free population structures (high $r$, low $\gamma$ ) (Payne and Eppstein 2009a). Since Gasparri et al. (2007, 2009) did not specify the algorithm used to construct their scale-free networks and did not provide any topological description of these networks, it is not possible to ascertain whether they fell into this specific subset or not. Relating experiment with theory is therefore not feasible in this case. Further, since the authors did not conduct any form of comparison with panmictic or lattice-based population structures, it cannot be said with certainty that scale-free population structures offered any real advantage in this application domain.

Kirley and Stewart (2007a) used genetic algorithms structured on random, small-world, and scale-free population structures for biobjective optimization. Six benchmark problems were analyzed and performance was measured in terms of convergence speed and the final spread of solutions across the Pareto front. Convergence time decreased as the average degree of the population structure increased, irrespective of any other topological properties. This result suggests that edge density may be the most important factor in determining the convergence rate of genetic algorithms embedded on complex population structures for biobjective optimization, an observation that could easily find theoretical support (Giacobini et al. 2005a; Lässig and Sudholt 2010; Payne and Eppstein 2009a; Sarma and De Jong 1996). When performance was measured in terms of the final spread of solutions across the Pareto front, scalefree population structures outperformed both a sparse small-world population structure and a regular population structure on the majority of benchmark problems. Despite this observation, Kirley and Stewart (2007a) concluded that the "indicator values for the scale-free network suggest that this particular architecture does not outperform the other network models." Even if their data supported this claim, their experimental design would preclude its validity. Only two of the six population structures considered possessed the same average degree, which makes it impossible to tease apart the effects of edge density from other topological properties, such as degree distribution. Thus, to conclude that scale-free population structures (which had the lowest average degree of any of the population structures tested) do not lead to performance improvements for biobjective optimization would require further support.

This support was provided in a follow-up study, where Kirley and Stewart (2007b) extended their analysis to include scale-free population structures with increased edge density and two benchmark multiobjective optimization problems with up to six objectives. Importantly, their revised experimental design allowed for the direct comparison of regular, small-world, and scale-free population structures of the same average degree $(z=8)$. Unfortunately, these results cannot be compared to those obtained with the random population structure because its average degree was $z \approx 50$. Focusing on the subset of results for which $z=8$, the performance differences obtained with various complex population structures were clearly problem dependent. In biobjective optimization, 
there was no significant difference in performance between regular, small-world, and scale-free population structures on the first benchmark problem. However, on the second benchmark problem, the small-world population structures outperformed both the regular and scale-free population structures. These observations support the authors' earlier claims that scale-free population structures are not advantageous in biobjective optimization (Kirley and Stewart 2007a). As the number of objectives increased, problem dependence persisted. On the first benchmark problem, scale-free population structures were outperformed by both small-world and regular population structures for three to six objectives. On the second benchmark problem, there was no significant difference between these three population structures in the majority of cases; the only exception was observed with six objectives, where scale-free population structures outperformed regular and small-world population structures. Thus, any performance improvements complex population structures might offer multiobjective optimization are clearly problem-dependent, echoing earlier results for single-objective optimization on regular population structures (Bryden et al. 2005).

\subsection{Open experimental questions}

The experimental studies discussed in the previous section have left many unanswered questions regarding the practical utility of complex population structures for evolutionary optimization. Two of the most pressing are listed here:

1. Are there specific classes of optimization problems that can benefit from small-world or scale-free population structures?

The above synthesis of existing experimental results suggests that the performance improvements offered by small-world and scale-free population structures are problem-dependent, as is also the case for regular population structures (Bryden et al. 2005). However, there may be some classes of optimization problems that can benefit from the use of complex population structures. It is unfortunately not possible to identify these classes from the existing literature, because the network construction methods, benchmark optimization problems, and algorithm parameters used in these studies are generally inconsistent and incomplete. A systematic analysis is therefore required that considers a more comprehensive range of small-world and scale-free population structures, a variety of evolutionary algorithm configurations, and a large suite of benchmark optimization problems. In so doing, it may be possible to identify the classes of optimization problems that can benefit from complex population structures.
2. How can the variability in selective pressure induced by scale-free population structures be exploited to enhance search performance?

In certain scale-free population structures, takeover times were highly variable, such that variability between initial placements was maximized on disassortative topologies, and variability between network instances was maximized on assortative topologies (Payne and Eppstein 2009a). It may be possible to capitalize on this variability by using scale-free population structures for (1) random restarts, a common (albeit crude) variation operator in which part or all of the population is randomly reinitialized (Fukunaga 1998); (2) an ensemble approach, in which multiple populations are evolved independently and a voting procedure is used to construct the final population (Breiman 1996); or (3) a metapopulation structure, in which the individual subpopulations are structured on scale-free networks.

\subsection{Future directions}

As outlined in the previous sections, several studies have investigated the effects of complex population structures on the performance of evolutionary optimization algorithms. However, these investigations have yet to be extended to complex metapopulation structures, wherein each vertex represents an entire subpopulation, instead of a single individual. In addition to the obvious extensions to random, small-world, and scale-free metapopulation structures, we outline below an alternative complex metapopulation structure that may be of interest for evolutionary computing.

Watts et al. (2005) proposed a hierarchical metapopulation structure to model resurgent epidemic outbreaks. The model is motivated by the observation that individuals form groups on multiple scales and that the strength of interaction between individuals decreases as the scale shifts from lower (e.g., family, workplace) to higher (e.g., town, country) levels.

This hierarchical structure is captured with the following formalism (Fig. 1). A population of $N$ individuals is evenly partitioned amongst $n$ well-mixed subpopulations. In each discrete time step, each individual migrates with probability $p$, transitioning from subpopulation $i$ to $j$ with probability $q_{i j} \propto \mathrm{e}^{x_{i j} / \xi}$. The hierarchy of subpopulations is thus encoded in the set $X=\left\{x_{i j}, \forall i, j\right\}$ and $\xi$ is a tunable parameter that determines the frequency of interaction between scales.

Analysis of the model's epidemic outbreaks (Watts et al. 2005) reveals three features of spreading dynamics that make this metapopulation structure appealing for evolutionary optimization. First, the duration of an outbreak is 


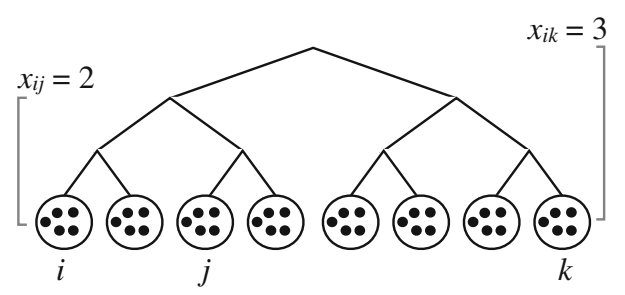

Fig. 1 Schematic of the hierarchical metapopulation model. Adapted from Watts et al. (2005)

significantly extended beyond what is expected in wellmixed populations. Second, the distribution of outbreak sizes is almost uniformly distributed, with the exception of a single peak near zero. Third, this distribution is relatively insensitive to changes in the basic reproduction number, a fundamental quantity in mathematical epidemiology that captures the number of secondary infections generated by a single infected individual (Falconer and Mackay 1996).

By drawing the analogy between an infection and an advantageous allele (Payne and Eppstein 2009b), the potential advantages of this metapopulation structure for evolutionary optimization become clear. Integrating the first two observations, this metapopulation structure clearly reduces selective pressure relative to the well-mixed case, but still allows for spreading events across all scales. In addition, the third observation implies that the distribution of spreading event sizes will be independent of the selective advantage of an allele, thus mitigating the problem of premature convergence. Importantly, selective pressure can be further tuned by adjusting $\xi$.

\section{Dynamic population structures}

\subsection{Synthesis of theoretical results}

Dynamic population structures are those that exhibit structural change throughout the evolution of the population. These population structures have received considerably less attention than their static counterparts. To date, only two studies (Alba and Dorronsoro 2005; Whitacre et al. 2008) have addressed the topic and both have focused on experimental results. As such, we currently do not possess any theoretical understanding of the very few dynamic population structures that have been introduced to the field.

\subsection{Open theoretical questions}

There are several open theoretical questions and outstanding challenges regarding dynamic population structures. A few are listed here:
1. How do dynamic population structures affect the fixation probabilities, dissemination, and mixing of advantageous alleles?

Extant dynamic population structures (Alba and Dorronsoro 2005; Whitacre et al. 2008) have no theoretical foundation, and the performance benefits they offer evolutionary optimization are therefore not well understood. An analysis of the influence of these dynamic population structures on the fixation probabilities, takeover times, and mixing of advantageous alleles would provide insight into how the various design choices adopted in these algorithms affect search performance.

2. Develop analytical models of takeover dynamics in dynamic population structures.

Analytical models of takeover dynamics, such as those developed for static regular lattices (Giacobini et al. 2005b), may provide further insight into the behavior of the dynamic population structures developed by Alba and Dorronsoro (2005) and Whitacre et al. (2008), particularly how they balance the explorative and exploitative components of evolutionary search. The inherent coupling of topological dynamics with population dynamics will necessitate a sophisticated modeling approach.

3. Can assortativity be tuned "on the fly" to adjust selective pressure in dynamic population structures?

Selective pressure and assortativity $(r)$ are inversely related in scale-free population structures, such that assortative topologies induce a low selective pressure (Payne and Eppstein 2008, 2009a). The assortativity of a population structure is also highly malleable, and can be changed with little overhead via simple edge swaps that maintain the degree distribution exactly. Thus, it may be possible to adjust selective pressure "on the fly" in response to various measures of population dynamics. For example, an initial study could focus on takeover time analysis, where assortativity changes in response to the gradient of the takeover curve. When the curve is steep, the topology adapts by becoming more assortative, and vice versa.

\subsection{Synthesis of experimental results}

As previously mentioned, selective pressure in regular population structures is a function of the ratio between the radii of the local neighborhood and the global lattice (Sarma and De Jong 1996). This observation was exploited by Alba and Dorronsoro (2005) to develop the first dynamic population structure for evolutionary optimization. The stated goal of this dynamic population structure was to adaptively balance the explorative and exploitative 
components of evolutionary search. To do so, the shape of the lattice was changed between square and rectangular forms in response to various measures of population dynamics, such as average fitness and population entropy. For example, if the population entropy decreased beyond some threshold between subsequent generations, then the shape of the lattice was made more narrow, reducing the ratio of the radii and increasing the population's explorative capacities.

This dynamic population structure was tested on a variety of benchmark optimization problems and compared to panmictic and static cellular evolutionary algorithms. In the majority of cases, the dynamic population structure outperformed the static population structures, both in terms of convergence time and percentage of successful trials.

Whitacre et al. (2008) adapted a model of genome duplication and divergence (Wagner 2001) as a dynamic population structure for mutation-based evolutionary optimization. The stated goal of this dynamic population structure was to recreate the structural characteristics of natural systems in an attempt to improve the search capabilities of evolutionary algorithms. The population structure was initialized as a ring, and the coupled dynamics of the population and population structure were separated into two distinct phases per generation. The first phase doubled the population size, using probabilistic rules for edge addition and deletion to incorporate new offspring. The second phase pared the population back down to its original size through local, winner-take-all competition events. Each birth and death event altered the population structure, transforming it away from its initial regular form toward an interaction network that possessed some of the topological features of natural populations, such as a heavy-tailed degree distribution.

This dynamic population structure was tested on NK landscapes (Kauffman 1993) with varying degrees of epistasis and was found to consistently outperform both panmictic and static ring-based CEAs. These performance improvements were attributed to enhanced diversity maintenance, with reported diversity levels increasing by approximately $20 \%$ over a ring-based CEA and $50 \%$ over a panmictic population. Impressively, these increases were even present amongst the individuals with top fitness scores, indicating that a variety of high-quality solutions were maintained in the population. A follow-up study by Payne and Moore (2010) investigated the effects of recombination in this dynamic population structure and showed that recombination leads to improved solution quality in deceptive problem domains, but offers no advantages in epistatic problem domains.

Dynamic population structures can also be found in Peerto-Peer evolutionary algorithms (Wickramasinghe et al.
2007), which aim to completely decentralize calculations related to selection, mating, and replacement. Individuals use so-called "gossiping protocols" (Jelasity et al. 2005) to estimate global information about the population from only a small subset of individuals. Since both this subset and the population size change over time, the underlying population structure is implicitly dynamic. Analysis of the structural properties of these population structures have shown that they can tend toward the small-world (Laredo et al. 2010), with high clustering coefficients and low characteristic path lengths. Additionally, peer-to-peer evolutionary algorithms can offer comparable solution quality to panmictic evolutionary algorithms on some difficult optimization tasks (Laredo et al. 2008), but with less computational overhead and improved scalability (Laredo et al. 2010). It should be noted, however, that it is not known whether these advantages stem from the inherent dynamism of the population structure or from the algorithm's distributed nature and the simplicity of its gossiping protocol.

\subsection{Open experimental questions}

Due to the recency of this area of research, there are many open questions, particularly regarding alternative forms of dynamic population structures. Here we focus on open questions that pertain to the two existing dynamic population structures:

1. What are the relative roles of mutation and recombination in dynamic population structures?

Alba and Dorronsoro (2005) investigated an evolutionary algorithm that used both mutation and recombination, while Whitacre et al. (2008) investigated an evolutionary algorithm that used only mutation. In the former case, a comparative analysis with a mutation-limited algorithm may provide a deeper understanding of how the dynamic restructuring of neighborhoods affects the mixing of genetic material, providing further insight into the performance benefits achieved with this dynamic population structure. In the latter case, a comparative analysis with a recombinative evolutionary algorithm has already been performed (Payne and Moore 2010), demonstrating that recombination can improve solution quality in certain problem domains. However, the performance improvements originally obtained with this dynamic population structure were attributed to enhanced diversity maintenance (Whitacre et al. 2008), yet Payne and Moore (2010) did not provide any analysis of the impact of recombination on population diversity. Such an investigation should be carried out to gain a better understanding of when and why recombination improves search performance in this dynamic population structure. 
2. How well do the performance benefits achieved with existing dynamic population structures translate to other optimization problems?

While Alba and Dorronsoro (2005) examined the performance of their dynamic population structure on a broad suite of benchmark problems, Whitacre et al. (2008) only considered NK landscapes. An obvious extension would be to consider a full range of benchmark problems, so that the generality of the results reported by Whitacre et al. (2008) can be assessed.

\subsection{Future directions}

In each of the previously developed dynamic population structures (Alba and Dorronsoro 2005; Whitacre et al. 2008), the manner in which local neighborhoods were restructured over time was defined a priori. For example, Alba and Dorronsoro (2005) allowed the ratio of the radii between the local neighborhood and the global lattice to change, but restricted the geometry of the population structure to a rectangular form. In natural populations, such restrictions do not exist. Individuals build their network of interactions throughout their lifetimes, creating and severing ties based on a variety of factors, including perceived benefits (Delton et al. 2011), previous interactions (Riolo et al. 2001), and reputations (Fu et al. 2008).

Such "active linking" has been investigated in the context of the evolution of cooperation, using both theoretical models (Pacheco et al. 2008) and human experiments (Rand et al. 2009). For instance, Pacheco et al. (2008) allowed the lifetime of inter-individual interactions to change in proportion to their payoff in an evolutionary game; interactions that led to high payoffs were more likely to persist than those that led to low payoffs. Rand et al. (2009) gave human subjects the opportunity to reward or punish other subjects for their behavior in a public goods game. Both of these theoretical and experimental studies found that active linking promotes the evolution of cooperation.

Active linking could be easily incorporated into spatially-structured evolutionary algorithms. For example, weights could be assigned to edges that determine the probability of interaction between connected individuals. The population could then evolve according to the standard update rules of cellular evolutionary algorithms, with the exception that the probability of an interaction is proportional to the corresponding edge weight. After each interaction, the respective edge weight could be updated according to its outcome; if a pair of connected individuals produce an offspring with fitness that is equal to or exceeds either parent, then the edge weight could be incremented, otherwise decremented. A potential starting point for the creation of such an algorithm could be the modification of anisotropic (Simoncini et al. 2006), centric (Simoncini et al. 2009), or polynomial (Vatanutanon et al. 2011) selection techniques, such that the interaction weights are dynamically adjusted.

One potential advantage of active linking for spatiallystructured evolutionary algorithms is that it may lead to a form of implicit niching (Mahfoud 1995). As interactions are tested throughout the evolution of the population, those edges that produce maladaptive offspring will tend to be severed, while those that produce high-fitness offspring will tend to be fortified. This will lead to a self-organized fragmentation of the population into discrete clusters of genetically or phenotypically similar individuals. While this may improve diversity maintenance, some method of edge creation will have to be incorporated into the algorithm to avoid search stagnation and promote exploration.

\section{Outlook}

In synthesizing the experimental literature on complex population structures, a general trend of problem-dependence was found. While further theoretical and experimental work is required to draw this conclusion definitively, we believe that the future of CEAs lies in dynamic population structures. By expanding upon the pioneering work of Alba and Dorronsoro (2005) and Whitacre et al. (2008), it should be possible to design population structures that more rapidly respond to population dynamics, further enhancing the explorative and exploitative capabilities of evolutionary search.

Acknowledgments J.L.P. was supported by NIH Grant No. R25CA134286 and J.H.M. was supported by NIH Grants LM010098, LM009012, and AI59694.

\section{References}

Alba E, Dorronsoro B (2005) The exploration/exploitation tradeoff in dynamic cellular genetic algorithms. IEEE Trans Evol Comput 9:126-142

Alba E, Dorronsoro B (2008) Cellular genetic algorithms. Springer, Berlin

Alba E, Tomassini M (2002) Parallelism and evolutionary algorithms. IEEE Trans Evol Comput 6(5):443-462

Barabàsi AL, Albert R (1999) Emergence of scaling in random networks. Science 286:509-512

Boguñá M, Pastor-Satorras R, Vespignani A (2003) Absence ofepidemic threshold in scale-free networks with degree correlations. Phys Rev Lett 90:028701

Breiman L (1996) Bagging predictors. Mach Learn 24:123-140

Bryden KM, Ashlock D, Corns S, Wilson S (2005) Graph based evolutionary algorithms. IEEE Trans Evol Comput 10(5):550 567

Cohoon J, Hegde S, Martin W, Richards D (1987) Punctuated equilibria: a parallel genetic algorithm. In: Proceedings of the 
2nd international conference on genetic algorithms. L. Erlbaum Associates Inc., pp 148-154

DeFelice M, Meloni S, Panzieri S (2011) Effect of topology on diversity of spatially-structured evolutionary algorithms. In: Proceedings of the genetic and evolutionary computation conference. ACM Press, pp 1579-1585

Delton AW, Krasnow MM, Cosmides L, Tooby J (2011) Evolution of direct reciprocity under uncertainty can explain human generosity in one-shot encounters. Proc Natl Acad Sci 108:13335-13340

Dorronsoro B, Bouvry P (2012) Study of different small-world topology generation mechanisms for genetic algorithms. In: Proceedings of the IEEE world congress on computational intelligenc, pp 1-8

Erdős P, Rényi A (1959) On random graphs. Publ Math 6:290-297

Falconer DS, Mackay TFC (1996) Quantitative genetics. Pearson Education Limited, London

Foo C, Kirley M (2008) An analysis of the effects of clustering in graph-based evolutionary algorithms. In: Michalewicz $\mathrm{Z}$ et al (eds) Proceedings of the IEEE congress on evolutionary computation. IEEE Press, pp 2246-2253

Fu F, Hauert C, Nowak MA, Wang L (2008) Reputation-based partner choice promotes cooperation in social networks. Phys Rev E 78:026117

Fukunaga A. S (1998) Restart scheduling for genetic algorithms. In: Genetic algorithms: proceedings of the seventh international conference, pp 357-358

Gasparri A, Panzieri S, Pascucci F (2009) A spatially structured genetic algorithm for multi-robot localization. Intell Serv Robot 2:31-40

Gasparri A, Panzieri S, Pascucci F, Ulivi G (2007) A spatially structured genetic algorithm over complex networks for mobile robot localisation. In: Proceedings of the IEEE international conference on robotics and automation. IEEE Press, pp 4277-4282

Giacobini M, Preuss M, Tomassini M (2006) Effects of scale-free and small-world topologies on binary coded self-adaptive CEA. In: Gottlieb J, Raidl GR (eds) Evolutionary computation and combinatorial optimization. Springer, Heidelberg, pp 86-98

Giacobini M, Tomassini M, Tettamanzi A (2005a) Takeover times curves in random and small-world structured populations. In: Beyer HG, (ed) Proceedings of the genetic and evolutionary computation conference. ACM Press, New York, pp 1333-1340

Giacobini M, Tomassini M, Tettamanzi A, Alba E (2005b) Selection intensity in cellular evolutionary algorithms for regular lattices. IEEE Trans Evol Comput 9(5):489-505

Goldbeck J, Parsia B, and Hendler J (2003) Trust networks on the semantic web. In: Klusch M, Omicini A, Ossowski S, Laamanen $\mathrm{H}$ (eds) Cooperative information agents VII. Lecture notes in computer science, vol 2782, pp 238-249. Springer, Berlin

Goldberg D, Deb K (1991) A comparative analysis of selection schemes used in genetic algorithms. In: Rawlins G (ed) Foundations of genetic algorithms. Morgan Kaufmann, San Francisco, pp 69-93

Gorges-Schleuter M (1989) Asparagos an asynchronous parallel genetic optimization strategy. In: Proceedings of the 3rd International Conference on Genetic Algorithms. Morgan Kaufmann, San Francisco, CA, USA, pp 422-427

Grefenstette J (1981) Parallel adaptive algorithms for function optimization. Technical Report CS-81-19, Vanderbilt University

Holland JH (1975) Adaptation in natural and artificial systems. PhD thesis, University of Michigan

Jelasity M, Montresor A, Babaoglu O (2005) Gossip-based aggregation in large dynamic networks. ACM Trans Comput Syst 23(3): 219-252

Jieyu W, Xinyu S, Jinhang L, Gang H (2012) Scale-free properties of information flux networks in genetic algorithms. Phys A 391: $1692-1701$
Kauffman SA (1993) The origins of order: self-organization and selection in evolution. Oxford University Press, Oxford

Kirley M, Stewart R (2007a) An analysis of the effects of population structure on scalable multiobjective optimization problems. In: Thierens D (ed) Proceedings of the genetic and evolutionary computation conference. ACM Press, New York, pp 845-852

Kirley M, Stewart R (2007b) Multiobjective optimization on complex networks. In: Fourth international conference on evolutionary multi-criterion optimization. Lecture notes computer science, pp 81-95

Laredo JLJ, Eiben AE, van Steen M, Castillo PA, Mora AM, Merelo JJ (2008) P2P evolutionary algorithms: a suitable approach for tackling large instances in hard optimization problems. In: EuroPar. Lecture Notes Computer Science, pp 622-631

Laredo JLJ, Eiben AE, van Steen M, Merelo JJ (2010) EvAg: a scalable peer-to-peer evolutionary algorithm. Genetic Program Evolvable Mach 11:227-246

Lässig J, Sudholt D (2010) General scheme for analyzing running times of parallel evolutionary algorithms. In: Parallel problem solving from nature. Lecture notes computer science, pp 234 243

Lieberman E, Hauert C, Nowak MA (2001) Evolutionary dynamics on graphs. Nature 433:312-316

Liljeros F, Edling CR, Amaral LAN, Stanely HE, Åberg Y (2001) The web of human sexual contacts. Nature 411:907-908

Mahfoud SW (1995) Niching methods for genetic algorithms. PhD Thesis, Champaign, IL, USA

Manderick B, Spiessens P (1989) Fine-grained parallel genetic algorithms. In: Proceedings of the third international conference on Genetic algorithms. Morgan Kaufmann Publishers Inc, San Francisco, pp 428-433

Min Y, Jin X, Su X, Peng B (2006) Empirical analysis of the spatial genetic algorithm on small-world networks. In: Sixth international conference on computational science, vol 3993. Lecture notes computer science, pp 1032-1039

Newman M (2010) Networks: an introduction. Oxford University Press, Oxford

Newman MEJ (2002) Assortative mixing in networks. Phys Rev Lett 89 :

Newman MEJ (2003) The structure and function of complex networks. SIAM Rev 45(2):167-256

Onnela JP, Saramäki J, Hyvönen J, Szabò G, Lazer D, Kaski K, Kertèsz J, Barabàsi AL (2007) Structure and tie strengths in mobile communication networks. Proc Natl Acad Sci 104:73327336

Pacheco JM, Traulsen A, Ohtsuki H, Nowak MA (2008) Repeated games and direct reciprocity under active linking. $\mathrm{J}$ Theor Biol 250:723-731

Pastor-Satorras R, Vespignani A (2001) Epidemic spreading in scalefree networks. Phys Rev Lett 86(4):3200-3203

Payne JL, Dodds PS, Eppstein MJ (2009) Information cascades on degree-correlated random networks. Phys Rev E 80:026125

Payne JL, Eppstein MJ (2006) Emergent mating topologies in spatially structured genetic algorithms. In: Keijzer M (ed) Proceedings of the genetic and evolutionary computation conference. ACM Press, New York, pp 207-214

Payne JL, Eppstein MJ (2007) Takeover times on scale-free topologies. In: Thierens D (ed) Proceedings of the genetic and evolutionary computation conference. ACM Press, New York, pp 308-315

Payne JL, Eppstein MJ (2008) The influence of scaling and assortativity on takeover times in scale-free topologies. In: Keijzer M (ed) Proceedings of the genetic and evolutionary computation conference. ACM Press, New York, pp 241-248

Payne JL, Eppstein MJ (2009a) Evolutionary dynamics on scale-free interaction networks. IEEE Trans Evol Comput 13:895-912 
Payne JL, Eppstein MJ (2009b) Pair approximations of takeover dynamics in regular population structures. Evol Comput 17:203-229

Payne JL, Moore JH (2010) Sexual recombination in self-organizing interaction networks. In: Proceedings of the EvoComplex conference, vol 6024. Lecture notes in computer science. Springer, Berlin, pp 41-50

Rand DG, Dreber A, Ellingsen T, Fundenberg D, Nowak MA (2009) Positive interactions promote public cooperation. Science 325:1272-1275

Riolo RL, Cohen MD, Axelrod R (2001) Evolution of cooperation without reciprocity. Nature 414:441-443

Rong Z, Li X, Wang X (2007) Roles of mixing patterns in cooperation on a scale-free networked game. Phys Rev E 76:027101

Rudolph G (2000) On takeover times in spatially structured populations: array and ring. In: Lai KK, Katai O, Gen M, Lin B (eds) Proceedings of the second Asia-Pacific conference on genetic algorithms and applications, APGA-2000. Global Link Publishing Company, Hong Kong, pp 144-151

Rudolph G (2001) Takeover times of noisy non-generational selection rules that undo extinction. In: Kurkova V (ed) Proceedings of the fifth international conference on artificial neural networks and genetic algorithms. Springer, Heidelberg, pp 268-271

Santos FC, Pacheco JM (2005) Scale-free networks provide a unifying framework for the emergence of cooperation. Phys Rev Lett 95:098104

Sarma J, De Jong K (1996) An analysis of the effect of neighborhood size and shape on local selection algorithms. In: Voigt HM, Ebeling W, Rechenberg I, Schwefel HP (eds) Parallel problem solving from nature. Springer, Heidelberg, pp 236-244

Simoncini D, Verel S, Collard P, Clergue M (2006) Anisotropic selection in cellular genetic algorithms. In: Proceedings of the genetic and evolutionary computation conference. ACM Press, New York, pp 559-566

Simoncini D, Verel S, Collard P, Clergue M (2009) Centric selection: a way to tune the exploration/exploitation trade-off. In: Proceedings of the genetic and evolutionary computation conference. ACM Press, New York, pp 891-898

Sood V, Antal T, Redner S (2008) Voter models on heterogeneous networks. Phys Rev E 77:041121
Tanese R (1987) Parallel genetic algorithms for a hypercube. In: Proceedings of the 2nd international conference on genetic algorithms. L. Erlbaum Associates Inc, USA, pp 177-183

Thierens D, Goldberg DE (1993) Mixing in genetic algorithms. In: Proceedings of the 5th international conference on genetic algorithms. Morgan Kaufmann Publishers, San Francisco, pp 38-47

Tomassini M (2005) Spatially structured evolutionary algorithms: artificial evolution in space and time. Springer, Berlin

Vatanutanon J, Noman N, Iba H (2011) Polynomial selection scheme with dynamic parameter estimation in cellular genetic algorithms. In: Proceedings of the genetic and evolutionary computation conference. ACM Press, New York, pp 1171-1178

Wagner A (2001) The yeast protein interaction network evolves rapidly and contains few redundant duplicate genes. Mol Biol Evol 18:1283-1292

Watts DJ (2002) A simple model of global cascades on random networks. Proc Natl Acad Sci 99:5766

Watts DJ, Muhamad R, Medina DC, Dodds PS (2005) Multiscale, resurgent epidemics in a hierarchical metapopulation model. Proc Natl Acad Sci 102:11157-11162

Watts DJ, Strogatz SH (1998) Collective dynamics of small-world networks. Nature 393:440-442

Whigham PA, Dick G (2008a) Evolutionary dynamics for the spatial moran process. Genetic Program Evolvable Mach 12(2):220-230

Whigham PA, Dick G (2008b) Exploring the use of ancestry as a unified network model of finite population evolution. In: IEEE congress on evolutionary computation. IEEE, New York, pp 3734-3740

Whitacre JM, Sarker RA, Pham QT (2008) The self-organization of interaction networks for nature-inspired optimization. IEEE Trans Evol Comput 12:220-230

Whitacre JM, Sarker RA, Pham QT (2009) Making and breaking power laws in evolutionary algorithm population dynamics. Memet Comput 1:125-137

Wickramasinghe W, van Steen M, Eiben AE (2007) Peer-to-peer evolutionary algorithms with adaptive autonomous selection. In: Proceedings of the genetic and evolutionary computation conference. ACM Press, New York, pp 1460-1467 